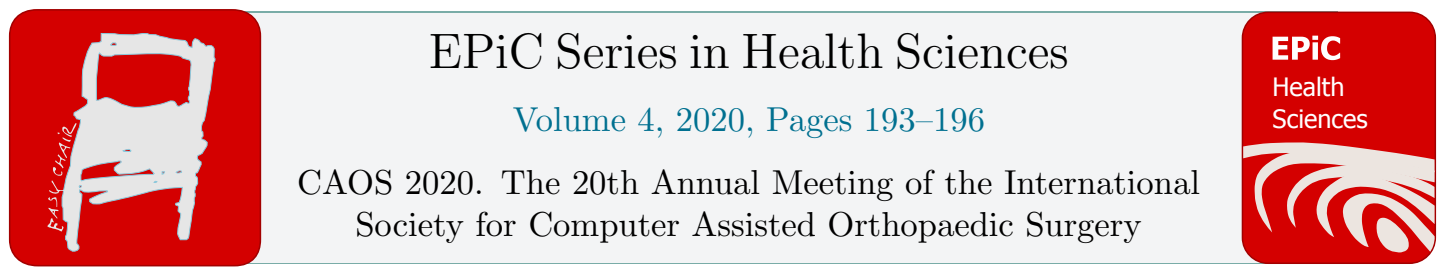

\title{
CAOS Augmented Mechanical Instrumentation Provides Versatility and Improved Accuracy during Total Knee Arthroplasty
}

\author{
Jefferson Craig Morrison1*, Erika Frazier2, Mary Stumb1 \\ 1*Southern Joint Replacement Institute, Tristar Centennial Hospital, Nashville, TN \\ 2 eMD Alliance Research, Franklin, TN \\ craig@sjri.com
}

\begin{abstract}
This study assessed the impact of adding computer-assisted orthopedic surgery (CAOS) augmentation to conventional mechanical instrumentation with regard to the enablement of both mechanical and natural alignment surgical philosophies and accuracy of postoperative alignment for total knee arthroplasty (TKA). Fifty CAOS augmented TKA cases were compared to 101 conventional cases. Data on surgical time, length of stay, and postoperative weight-bearing long leg alignment were collected. The results reported no significant impact on surgical time with incorporation of CAOS augmentation into the conventional surgical workflow, as well as a shorter length of stay and substantial decrease in alignment outliers compared to the conventional TKA cases. The study revealed the advantages of CAOS augmentation in providing a non-disruptive tool to enhance surgical accuracy and offer versatility in accommodating different surgical philosophies during TKA.
\end{abstract}

\section{Introduction}

Accurate positioning of the knee prosthesis is critical for the success of total knee arthroplasty (TKA) [1]. However, with mechanical (neutral) alignment surgical philosophy, only $70-80 \%$ of the conventional TKA cases can achieve satisfactory accuracy (within $\pm 3^{\circ}$ of varus/valgus relative to the mechanical axis) [2,3]. Furthermore, it still remains unsolved that up to $20 \%$ of patients are dissatisfied with their surgery [4]. To improve the outcome of TKA, emphasis has been made that a surgeon should minimize surgical errors that lead to malalignment. Furthermore, new surgical philosophies, such as natural alignment, have been applied as healthy knees demonstrates constitutional varus in up to $32 \%$ of adult men and $17 \%$ of adult women [5]. This philosophy proposed that restoration of a slight varus 
CAOS Augmented Mechanical Instrumentation Provides Versatility and Improved ... Morrison et al.

alignment in varus knees during total knee arthroplasty (TKA) may offer benefits to better postoperative natural kinematics [6]. However, in actual practices, it is a paramount technical challenge to determine and achieve a natural alignment using conventional TKA instruments with sufficient efficiency and accuracy.

Computer-assisted orthopedic surgery (CAOS) offers increased accuracy and precision to the bony resections compared to the conventional techniques [7]. This technology may offer a versatile tool to accommodate both mechanical and natural alignment surgical philosophies. However, one of the drawbacks for its adoption by the surgeons may be the inconvenience of switching from conventional instruments to CAOS-specific instruments. To remove the barrier to its adoption, a novel system has been introduced to augment conventional mechanical instruments with CAOS technology. In this study, the surgeon author sought to demonstrate and assess the impact of the CAOS augmentation on conventional instrumentation regarding resection accuracy and application of both surgical philosophies.

\section{Materials and Methods}

With IRB oversight and its determination of exemption according to 45 CFR 46.104 category $\# 4$, a retrospective consecutive TKA case series was conducted by the surgeon author. The series consisted of 50 cases performed with mechanical instrumentation augmented by CAOS, matched with 101 conventional cases performed prior to the series using the same mechanical instruments. All the conventional cases targeted mechanical alignment due to the difficulty and low reliability of applying target natural alignment amount. In the CAOS augmented cases, natural alignment (2-3 $3^{\circ}$ residual Varus) was targeted for preoperative alignment of more than $5^{\circ}$ varus. As such, the acceptable range for the natural alignment cases was set to be $0-5^{\circ}\left(2-3^{\circ} \pm 3^{\circ}\right)$, taking into account that in no cases the surgeon accepted more than $5^{\circ}$ varus in the final alignment. The rest of the cases aimed for mechanical alignment.

Surgical time, speed of recovery, and alignment outcomes were collected, including tourniquet time, length of stay (LOS), and long-leg weight-bearing coronal alignment (hip-knee-ankle angle) measured at 6-month postoperatively. Outliers in alignment were identified as hip-knee-ankle angle within $\pm 3^{\circ}$ regarding mechanical axis for the mechanically aligned cases, and within $0^{\circ}-5^{\circ}$ varus for the naturally aligned cases. The results were compared between the conventional and CAOS augmented groups. Statistical significance was defined as $\mathrm{p}<0.05$.

\section{Results}

No significant difference was found in the patients' age and BMI between the CAOS augmented group and the conventional group ( $\mathrm{p}$ values $\geq 0.40$ ) (Table 1). Preoperatively, the CAOS augmented group was on average more varus then the conventional group (Table 1).

Among the 50 CAOS augmented cases, 27 were performed under natural alignment philosophy as thy exhibited preoperative varus alignment of more than $5^{\circ}$ (ranged from $6^{\circ}$ to $15^{\circ}$ ). For both mechanically and naturally aligned groups, CAOS augmentation of the mechanical instrumentation did not increase tourniquet time from the conventional group ( $\mathrm{p}>0.04)(\mathrm{Fig} 1 \mathrm{~A}, \mathrm{~B})$. The CAOS augmented patients had significantly shorter LOS (by 0.4 day) than the conventional patients (Fig $1 \mathrm{C}, \mathrm{p}=0.02$ ). The use of CAOS augmentation exhibited significantly lower alignment outlier rate (2.4\%) compared to the conventional cases $(30.0 \%)(\mathrm{p}<0.01)$ (Fig 1D). 
CAOS Augmented Mechanical Instrumentation Provides Versatility and Improved ... Morrison et al.

\section{Discussion}

This study reported no increase in surgical time, shorter length of stay, and significantly reduced postoperative alignment outliers with the introduction of CAOS augmentation to conventional mechanical instrumentation during TKA. Furthermore, the technology enabled the application of natural alignment surgical philosophy in the varus knees. In contrast, conventional instruments do not provide a way to measure and set patient-specific alignment target. Even if the natural alignment target may be defined by specially designed conventional instrumentation, the inherit variability from the use of such instrumentation may be significant enough to impede a surgeon's ability to achieve a predefined natural alignment value. It has been shown that approximately $30 \%$ of the TKA cases using conventional instrumentation had alignment errors exceeding $3^{\circ}[8,9]$. The CAOS augmentation greatly facilitated the surgical technique by offering the ability to quantify the alignment target, provide guidance to bony resection, and assess the accuracy of the achieved alignment.

In conclusion, the study demonstrated that augmenting conventional mechanical instrumentation with CAOS technology can be advantageous in achieving improved alignment accuracy, speed of recovery, and offer surgeons the versatility in applying preferred alignment surgical philosophy without the compromise of surgical time. Future follow up of the patients' outcome may provide further assessment of the CAOS assisted naturally aligned TKA.

Table 1. A summary of patient demographics and preoperative alignment in the CAOS augmented and conventional groups.

\begin{tabular}{lcccc}
\hline Instrumentation & $\mathbf{N}$ & Age $(\mathbf{y r})$ & $\mathbf{B M I}$ & $\mathbf{H K A}^{\star}\left({ }^{\circ}\right)$ \\
Conventional & 101 & $70.2 \pm 9.2$ & $30.5 \pm 5.2$ & $-1.1 \pm 5.8$ \\
CAOS Augmented & 50 & $67.3 \pm 7.8$ & $31.2 \pm 4.6$ & $4.5 \pm 6.7$ \\
$\quad$ Preop Varus $>5^{\circ}$ & 27 & $66.7 \pm 8.1$ & $31.5 \pm 4.6$ & $9.6 \pm 2.6$ \\
Other & 23 & $67.9 \pm 7.6$ & $31.0 \pm 4.6$ & $-1.4 \pm 4.9$ \\
\hline
\end{tabular}

*A positive value indicates varus alignment.
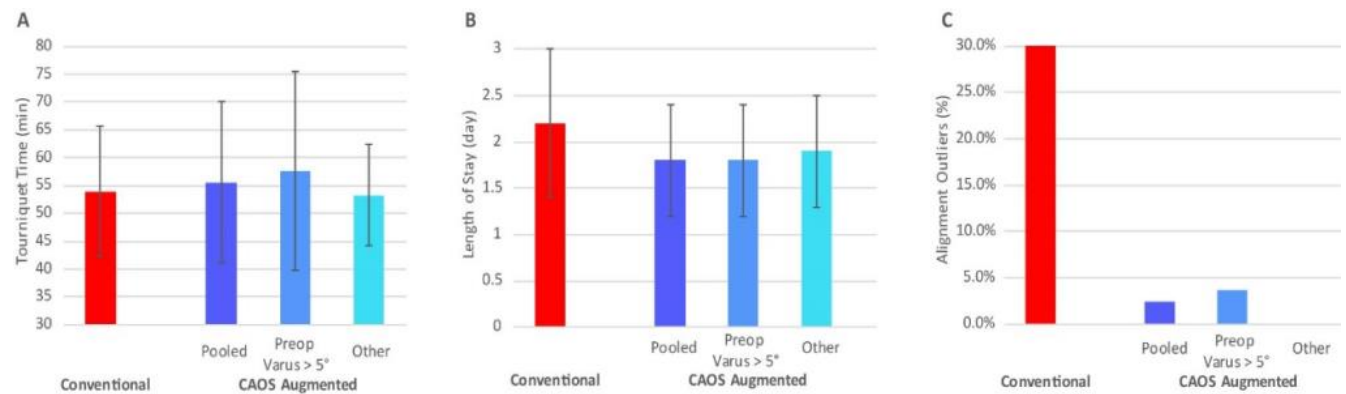

Figure 1. Comparison of A) tourniquet time, B) length of stay, and C) alignment outlier \% between the conventional group and CAOS augmented group. 
CAOS Augmented Mechanical Instrumentation Provides Versatility and Improved ... Morrison et al.

\section{References}

[1] Fehring TK, Odum et al. Clin Orthop Relat Res.2001;(392):315-8.

[2] Ensini A, et al. Clin Orthop Relat Res. 2007;457:156-62.

[3] Hetaimish BM, et al. J Arthroplasty. 2012;27(6):1177-82.

[4] Scott CE, et al. J Bone Joint Surg Br. 2010;92(9):1253-8.

[5] Bellemans J, et al. Clin Orthop Relat Res, 470(1):45-53, 2012.

[6]Schiffner E, et al. J Knee Surg. 2019 Aug;32(8):820-824.

[7] Sparmann M, et al. J Bone Joint Surg Br. 2003;85(6):830-5.

[8] Petersen TL, et al. J Arthroplasty, 3:67-72, 1988.

[9] Mahaluxmivala J, et al. J Arthroplasty,16:635-40, 2001. 